\title{
Research Paper \\ Effects of a Combined Aerobic and Resistant Exercise Training Program on Blood Pressure and Heart Rate Variability in Pregnant Women: A Randomized Clinical Trial
}

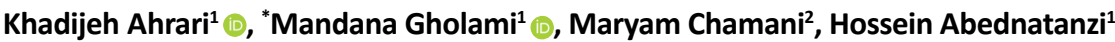

1. Department of Physical Education \& Sport Sciences, Faculty of Literature, Humanities and Social Sciences, Science and Research Branch, Islamic Azad University Tehran, Iran.

2. Department of Obstetrics and Gynecology, Shahid Akbarabadi Hospital, Clinical Research Development Unit (ShAcrdu), Iran University of Medical Sciences, Tehran, Iran.

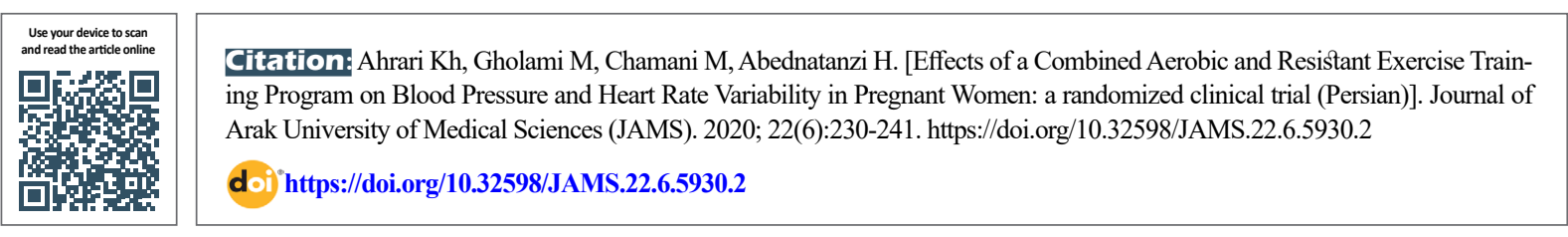

\section{(c) (i) (8)}

Article Info:

Received: 25 Oct 2019 Accepted: 28 Dec 2019 Available Online: 01 Feb 2020

Key words:

Combined exercise training, Blood pressure, Heart rate variability, Pregnancy

\section{ABSTRACT}

Background and Aim Pregnancy and exercise training are associated with changes in the cardiovascular anatomy and physiology. There is a few studies on this issue in Iran. In this regard, the aim of this study is to assess the effect of a combined exercise training program on blood pressure and heart rate variability of pregnant women.

Methods \& Materials This randomized clinical trial was conducted in Shahid Akbarabadi Hospital in Tehran Iran in 2019. Participants were 10 healthy pregnant women with a gestational age of 24-26 weeks in the interventional group and 10 healthy pregnant women in the control group. The interventional group received 8 weeks of combined exercise training at moderate intensity. The blood pressure measurement, electrocardiogram and aerobic and resistant exercise tests of samples were performed before and after training. AST3000 Avicenna software was used to analyze the heart rate variability parameters, and for statistical analysis, paired and independent t-tests, Mann Whitney $U$, and Wilcoxon tests were used.

Ethical Considerations This study with registered clinical trial Code IRCT20190227042856N1 was approved by the Research Ethics Committee of Islamic Azad University of Science and Research Branch in Tehran, Iran (Code: IR.IAU.SRB.REC.1397.101).

Results The mean systolic and diastolic blood pressure levels were lower in the interventional group by the $34^{\text {th }}$ week of pregnancy compared to the control group $(P<0.05)$. The mean of time-domain parameters in the interventional group were higher by the $34^{\text {th }}$ week of pregnancy compared to the control group $(P<0.05)$. Conclusion Combined exercise training can improve autonomic and parasympathetic nervous systems in pregnant women.

\section{Extended Abstract}

\section{Introduction}

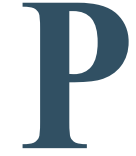

regnancy and exercise training are associated with changes in the cardiovascular anatomy and physiology. Today, the demand of pregnant women to participate in exercise activities has increased, and various studies have emphasized the combination of different types of exercises, especially aerobic and resistance exercises, to obtain better results [3]. The effect of exercise on the cardiovascular system of the mother and fetus according to their different protocols and the main criteria of prescribing exercise has not been adequately investigated with appropri-

\section{* Corresponding Author:}

Mandana Gholami, PhD.

Address: Department of Physical Education \& Sport Sciences, Faculty of Literature, Humanities and Social Sciences, Science and Research Branch, Islamic Azad University, Tehran, Iran.

Tel: +98 (21) 44861790

E-mail:m.gholami@srbiau.ac.ir 
ate methodology in the world and especially in Iran. Moreover, in previous limited studies, contradictory results have been reported on cardiovascular hemodynamic changes and parameters of maternal and fetal heart rate variability following exercise training $[5,6]$. In this regard, this study aimed to investigate the effect of a combined aerobic and resistance exercises training program on blood pressure and heart rate variability in pregnant women.

\section{Methods and Materials}

This study is a single-blind, parallel-group, two-stage randomized clinical trial. The study population consisted of all healthy pregnant women referred to Shahid Akbarabadi Hospital in Tehran, Iran in 2019. Inclusion criteria for participants were: Age 20 to 35 years, singleton pregnancy, body mass index of 18.5-25, pregnancy frequency less than 5, and blood pressure $<140.90$. Cardiovascular and orthopedic diseases, pregnancy complications and having a history of high risk pregnancy were exclusion criteria. Based on these criteria, 20 samples were selected and randomly divided into two groups of exercise $(n=10)$ and control $(n=10)$. The exercise group received combined exercise training for 8 weeks, 3 sessions per week at moderate intensity from the week 24-26 of gestation. Exercise tests, anthropometric measurements, blood pressure, and electrocardiogram test were performed before and after intervention. AST3000 Avicenna software was used to analyze the heart rate variability parameters. For statistical analysis, paired and independent t-tests, Mann Whitney U, Fisher's test, Shapiro-Wilk test and Wilcoxon tests were used in SPSS V. 24 sofwtare.

\section{Results}

According to the findings, there was no statistically significant difference in age, height, weight, body mass index, body fat percentage, maximum oxygen consumption, education, number of deliveries, and physical activity between the exercise and control groups ( $\mathrm{P}>0.05)$. Before combined exercise, resting heart rate, systolic and diastolic blood pressures were similar in the two groups $(\mathrm{P}>0.05)$, but the mean of these variables were significantly lower in the exercise group after eight weeks of combined exercise training $(\mathrm{P}<0.05)$. Moreover, in both groups, the mean diastolic and systolic blood pressures were significantly higher at 34-36 weeks of gestation than at 24-26 weeks $(\mathrm{P}<0.05)$ (Table 1). Comparing the results of the exercise group before and after intervention, the Standard Deviation of NN intervals (SDNN) in pregnant women at 34-36 weeks of gestation was higher than that at 24-26 weeks of gestation $(\mathrm{P}<0.05)$. The mean of time-do-

Table 1. Heart rate and blood pressure level of participants

\begin{tabular}{|c|c|c|c|c|c|c|}
\hline \multirow{3}{*}{ Variabels } & \multicolumn{4}{|c|}{ Mean $\pm S D$} & \multirow{2}{*}{\multicolumn{2}{|c|}{ Sig. }} \\
\hline & \multicolumn{2}{|c|}{ Exercise Group } & \multicolumn{2}{|c|}{ Control Group } & & \\
\hline & 24-26 Weeks & 34-36 Week & 24-26 Weeks & 34-36 Week & $\begin{array}{c}\text { Pre-test/ Post-test } \\
\text { Scores }\end{array}$ & Control/Exercise Group \\
\hline Heart rate (bpm) & $78.60 \pm 3.47$ & $80 \pm 3.16$ & $81.90 \pm 4.56$ & $82.90 \pm 3.18$ & $* 0.05$ & $* * 0.05$ \\
\hline $\begin{array}{c}\text { Diastolic blood } \\
\text { pressure (MmHg) }\end{array}$ & $67.90 \pm 5.24$ & $72.90 \pm 3.96$ & $73.40 \pm 6.88$ & $78.1 \pm 3.57$ & $* * * *<0.001$ & $* * * 0.007$ \\
\hline $\begin{array}{c}\text { Systolic blood } \\
\text { pressure (MmHg) }\end{array}$ & $110.10 \pm 5.69$ & $113.70 \pm 4.92$ & $110.5 \pm 6.93$ & $118.30 \pm 3.47$ & $* * * *<0.001$ & $* * * 0.031$ \\
\hline
\end{tabular}

*Paired t-test; ${ }^{* *}$ Independent t-test; $* * *$ Wilcoxon test

Table 2. Time-domain parameters of heart rate variability in participants at 34-36 weeks of gestation

\begin{tabular}{|c|c|c|c|}
\hline \multirow[b]{2}{*}{ Parameter } & \multicolumn{2}{|c|}{ Mean $\pm S D$} & \multirow{2}{*}{ Sig.* } \\
\hline & $\begin{array}{l}\text { Exercise Group } \\
\text { 34-36 Weeks }\end{array}$ & $\begin{array}{c}\text { Control Group } \\
34-36\end{array}$ & \\
\hline SDNN (ms) & $74.2 \pm 7.22$ & $51.80 \pm 13.87$ & 0.001 \\
\hline RMSSD (ms) & $48.10 \pm 27.61$ & $27.70 \pm 31.01$ & 0.04 \\
\hline
\end{tabular}

* Mann Whitney U test 
Table 3. Frequency-domain parameters of heart rate variability in participants at 34-36 weeks of gestation

\begin{tabular}{|c|c|c|c|}
\hline \multirow[b]{2}{*}{ Parameters } & \multicolumn{2}{|c|}{ Mean \pm SD } & \multirow[b]{2}{*}{ Sig.* } \\
\hline & $\begin{array}{l}\text { Exercise Group } \\
\text { 34-36 weeks }\end{array}$ & $\begin{array}{c}\text { Control Group } \\
34-36\end{array}$ & \\
\hline $\operatorname{LF}\left(\mathrm{ms}^{2}\right)$ & $251.60 \pm 362.15$ & $126.70 \pm 152.82$ & 0.97 \\
\hline $\mathrm{HF}\left(\mathrm{ms}^{2}\right)$ & $200.90 \pm 296.59$ & $124.50 \pm 105.45$ & 0.99 \\
\hline LF.HF & $2.08 \pm 2.08$ & $1.78 \pm 2.21$ & 0.45 \\
\hline
\end{tabular}

main parameters including SDNN and Root Mean Square of Successive Differences (RMSSD) in the exercise group were higher at the 34th week of pregnancy compared to the control group $(\mathrm{P}<0.05)$ (Tables 2 and 3$)$.

\section{Discussion}

Based on the findings of this study, the mean of heart rate, systolic blood pressure and diastolic blood pressure in pregnant women received 8 weeks of combined exercise were lower than in controls. Decreased vasomotor tone, increased parasympathetic neural activity, and mediators such as nitric oxide following exercise may be the reasons for lower blood pressure and heart rate in the exercise group than in the control group [15].

Increased SDNN parameter in the exercise group compared to the control group indicates that combined exercise improves the autonomic nervous system balance. However, in pregnancy, the parameters of heart rate variability decrease or do not change with increasing gestational age and increasing weight of pregnant women [4]. In this study, the RSMMD parameter was higher in trained pregnant women and in 34-36 weeks of pregnancy compared to the control group. This difference was statistically significant. In other words, combined exercise resulted in the relatively high parasympathetic nerve activity in the exercise group [5]. May et al. also reported the mean increase in heart rate variability of pregnant women performed exercise training [5, 20]. Their study was a case-control study, so there was a possibility of confounding effects. In Satyapriya et al.'s study, two groups of pregnant women with different exercises were studied. In two groups, HF increased and LF and LF / HF decreased; however, no control group was included in their study [22]. The reason for the discrepancies between the results of this study and others can be the difference in the number of samples, the order of exercise, body weight, and gestational age $[5,6]$.

\section{Conclusion}

The combined exercise training group can improve heart rate, blood pressure and heart rate variability in pregnant women. Further studies are recommended to investigate the effects of combined exercises with different intensity and the mechanism of their effects on blood pressure and heart rate variability.

\section{Ethical Considerations}

\section{Compliance with ethical guidelines}

This study with registered clinical trial code IRCT20190227042856N1 was approved by the Research Ethics Committee of Islamic Azad University of Science and Research Branch in Tehran, Iran (Code: IR.IAU.SRB.REC.1397.101).

\section{Funding}

This study was extracted from thesis of the first author, Khadijeh Ahrari, approved by the Department of Physical Education and Sports Science, Islamic Azad University, Science and Research Branch.

\section{Authors' contributions}

All authors met the standards of writing based on the recommendations of the International Committee of Medical Journal Editors (ICMJE).

\section{Conflicts of interest}

The authors declare no conflict of interest.

\section{Acknowledgements}

The authors would like to thank the faculty professors, the staff of Perinatology Clinic and Clinical Research Development Unit of Shahid Akbarabadi Hospital in Tehran. 


\title{
تاثير تمرين تركيبى هوازى و مقاومتى برفشار خون و تغييريذيرى ضربان قلب در زنان باردار: يكى مطالعه كار آزماييى بالينى تركيى تمائري
}

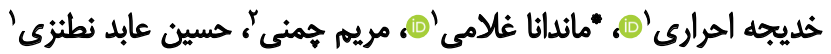 \\ 1. كروه تربيت بدنى وعلوم ورزشى، دانشكده ادبيات، علوم انسانى واجتماعى، دانشكاه ازإد اسلامى، واحد علوم تحقيقات، تهران، ايران.

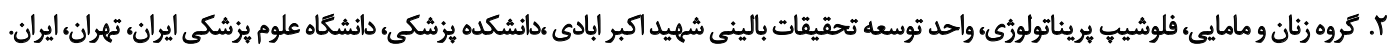

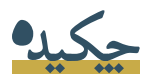

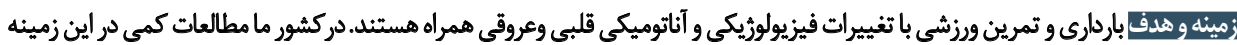

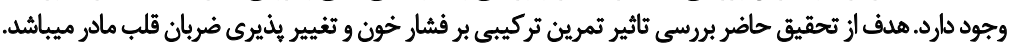

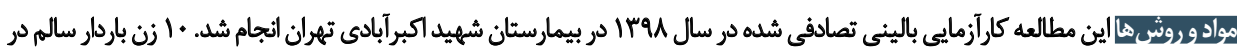

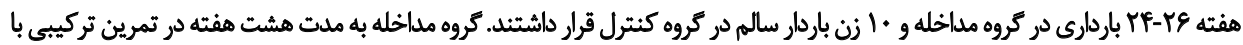

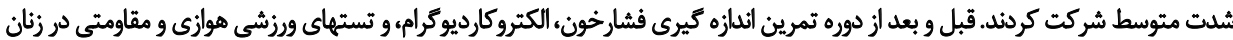

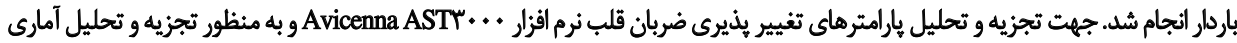

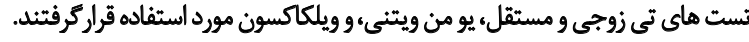

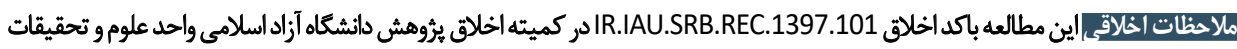

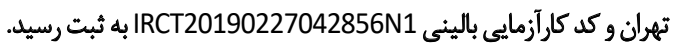

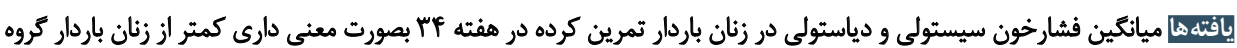

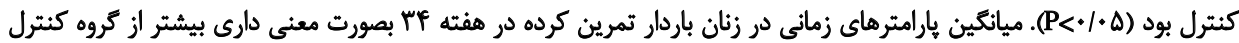
.

تتيجه كيرى تمرين تركيبى ميتواند موجب بهبود سيستم عصبى خود مختار قلبى و برترى سيستم عصبى باراسمباتيك در زنان باردار شود.
\end{abstract}

اطلاعات مقاله: تاريخ دريافت: ب. آبان

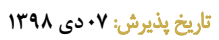

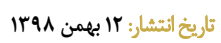

كليدوأرهها: تمرين ورزشى تركيبى،

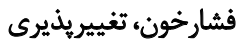

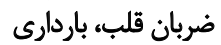

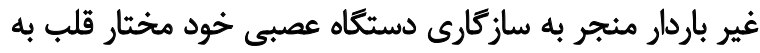

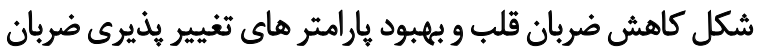

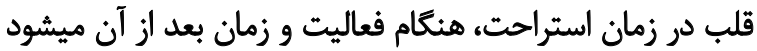

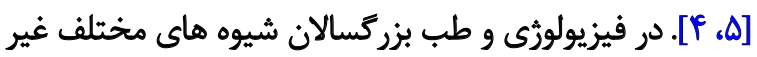

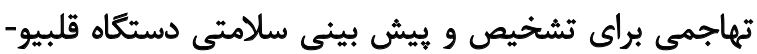

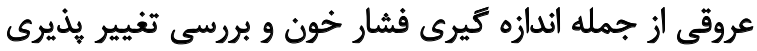

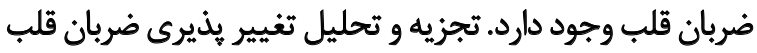

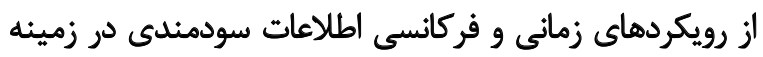

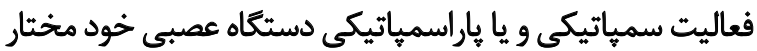

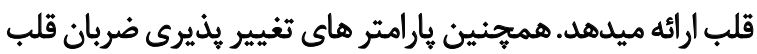

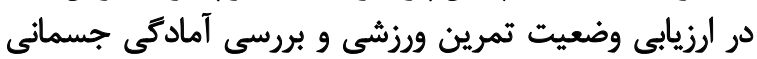

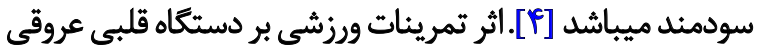

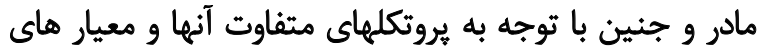

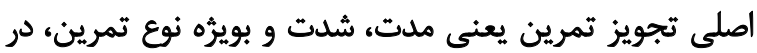

doles

تمرينات ورزشى بدليل تغييرات فيزيولوزيكى مناسب، اثرات

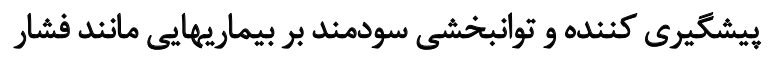

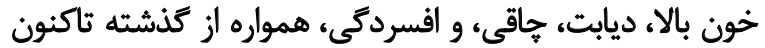

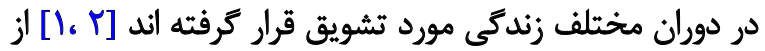

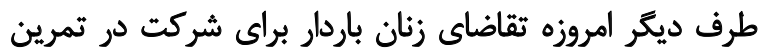

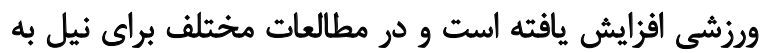

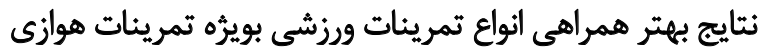

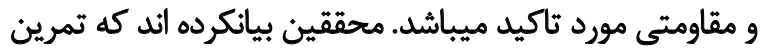

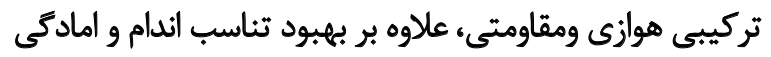

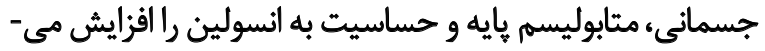

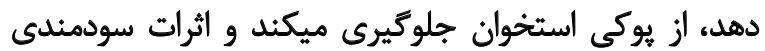

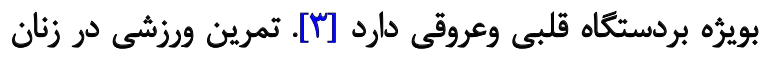

* نويسنده مسئول:

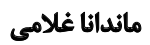
نشائى: تهران، واحد علوم و تحقيقات، دانشكاه آزاد اسلامي، دانشكده ادبيات علوم انسانى و اجتماعى، كروه تربيت بلدنى و علوم ورزشى.

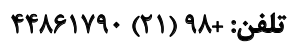
m.gholami@srbiau.ac.ir يست الكترونيكى 
جلسات تمرين شركت كردند و تمام بررسيهاى مربوط به اهداف

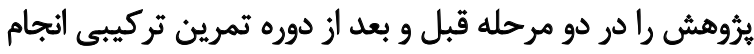

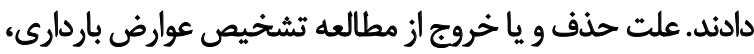

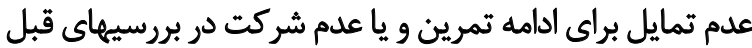

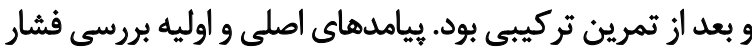

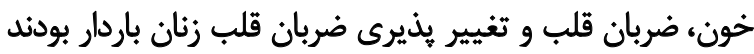

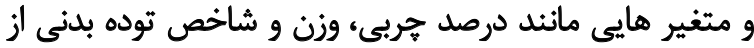

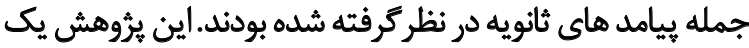

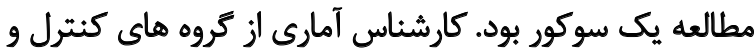

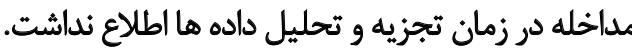
در اين مطالعه كه در بيمارستان شهيد اكبرآبادى تهران انجام

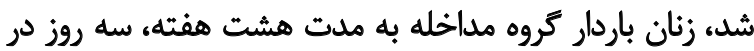

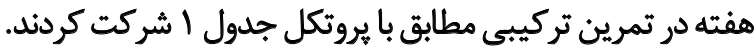

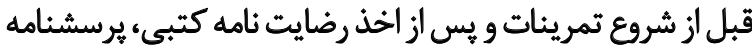

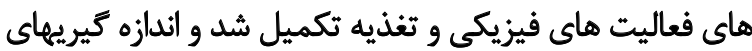

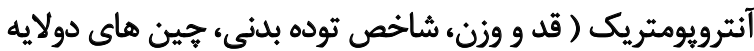

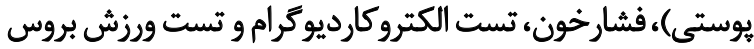

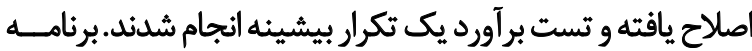

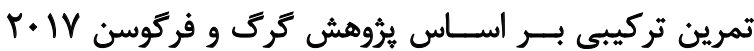

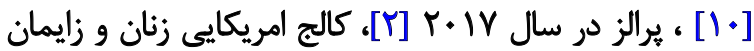

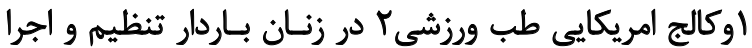

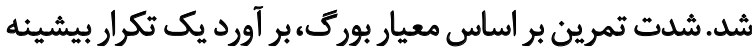

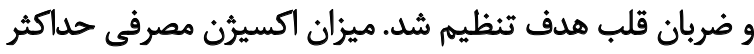

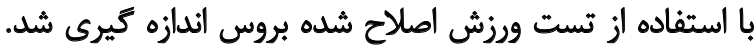

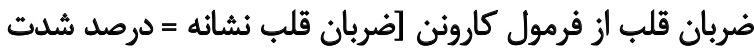

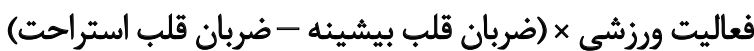

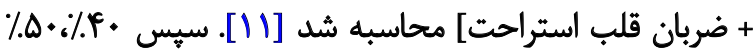

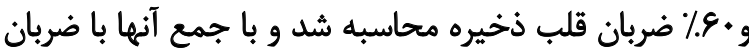

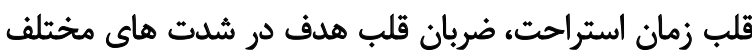
تمرين هوازى تنظيم كرديد.

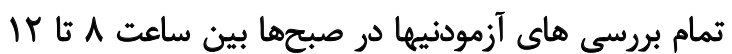

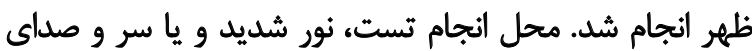

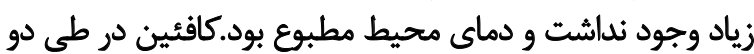

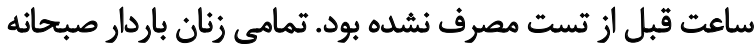

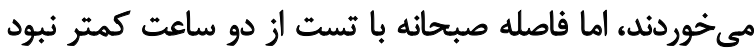

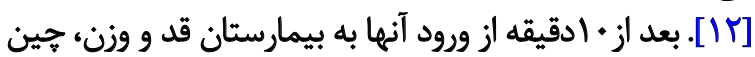

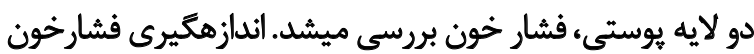

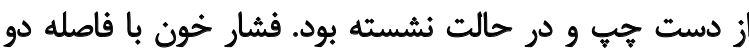

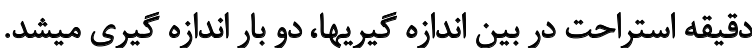

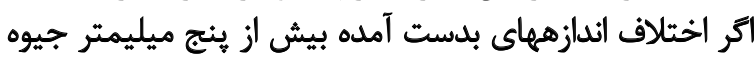

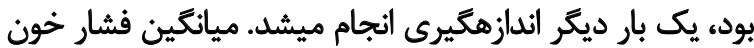

1. American college of obstetrics and gynecology

2. American college of sports medicine
جهان و بطور خاص در كشور ما به ميزان كافى و با متدولوزى

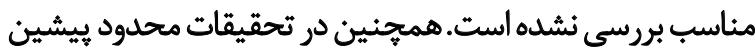

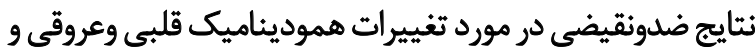

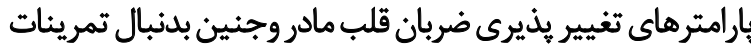

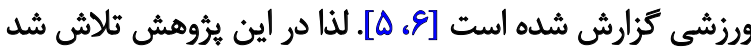

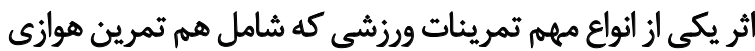

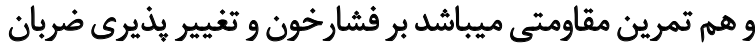
قلب زنان باردار بررسى تردد.

\section{مواد و روش ها - ماث}

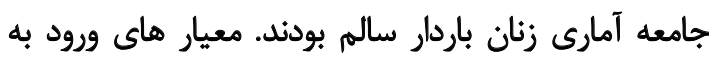

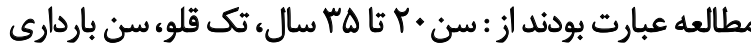

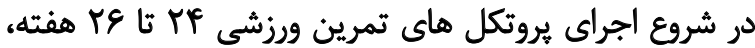

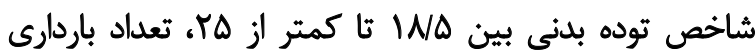

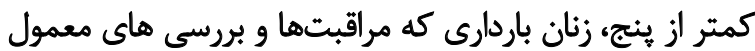

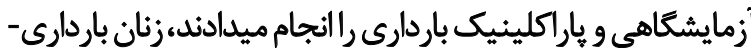

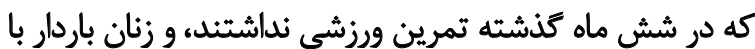

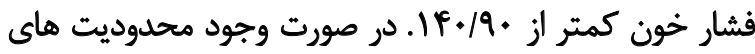

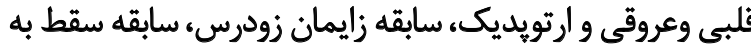

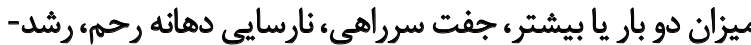

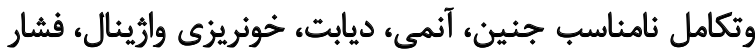

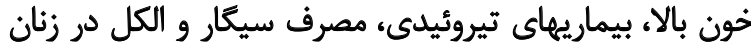

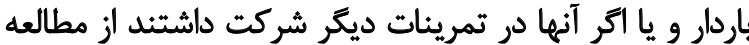

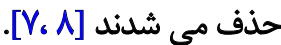

تمامى زنان باردار تحت مراقبت هاى روتين بارئ باردارى قرار

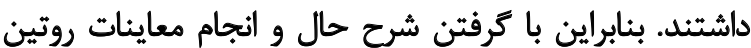

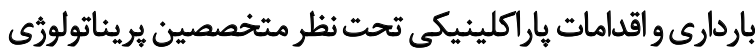

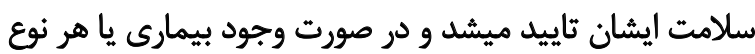
عارضه باردارى از مطالعه حذف ميشداند. حجم نموند

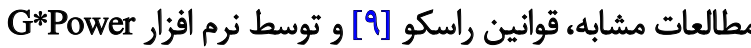

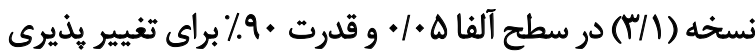

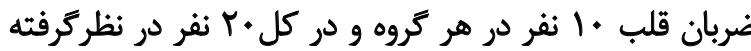

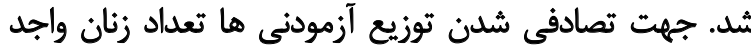

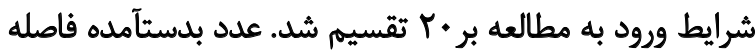

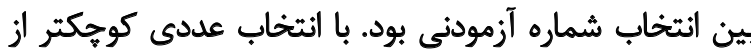

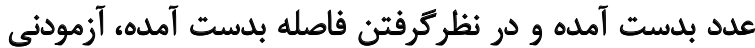

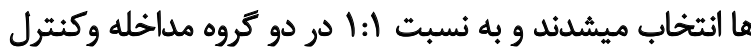

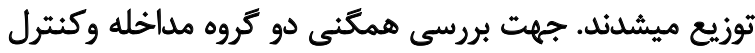

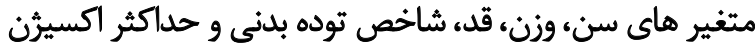

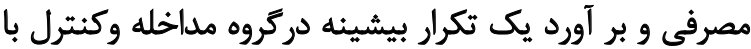

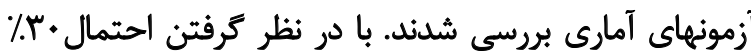

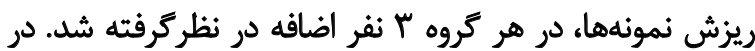

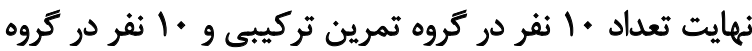

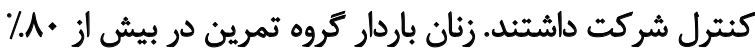


اقدامات آزمايشعاهي و ياراكلينيكى مورد تاييد اساتيد دانشكاه

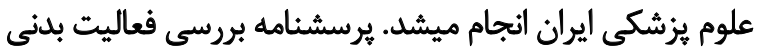

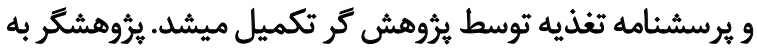

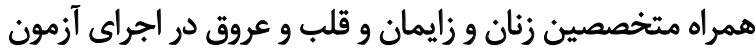

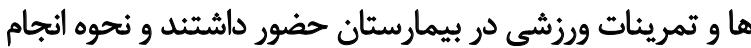

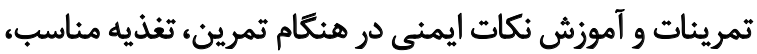

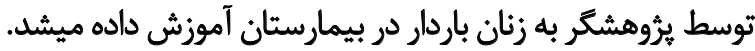

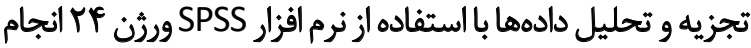

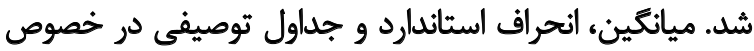

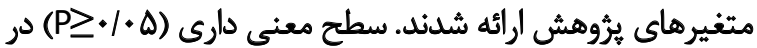

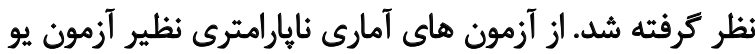

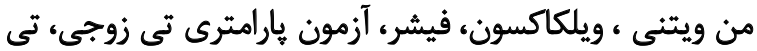
مستقل و آزمون شايِيروويك استفاده شد.

L) L

ويرُكيهاى آزمودنيها، در جداول بو بَ آورده شده اند. بر اساس

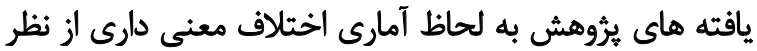

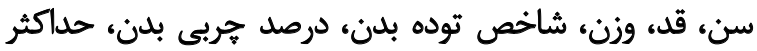

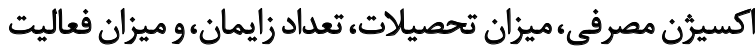

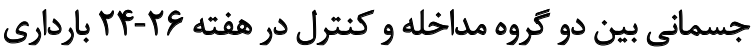

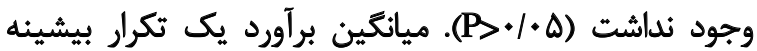

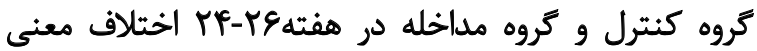

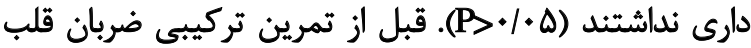

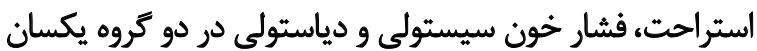

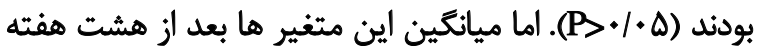

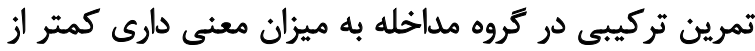

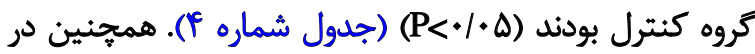

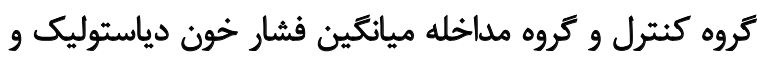

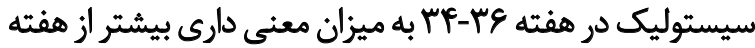

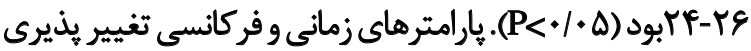

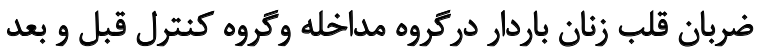

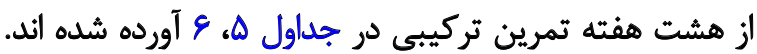

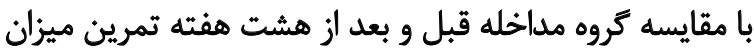

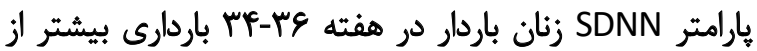

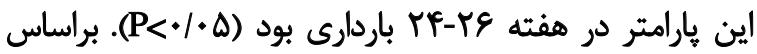

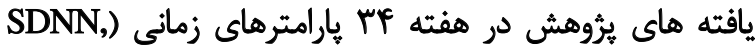
(RMSSD

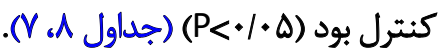

بحث

بر اساس يافته هاى يُؤهش حاضر ميانكين ضربان قلب فشار

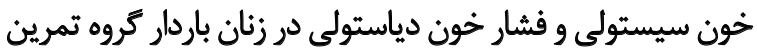

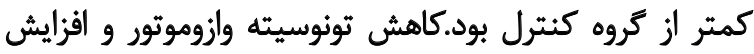

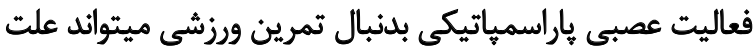

دو اندازه بدست آمده به عنوان فشارخون آزمودنى در نظر كرفته

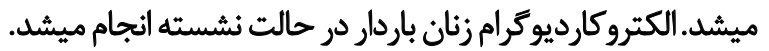

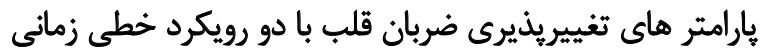

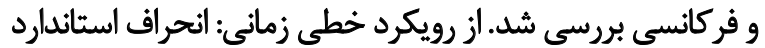

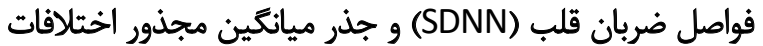

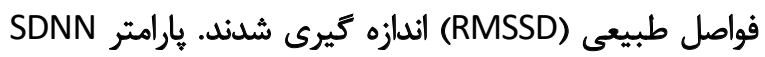

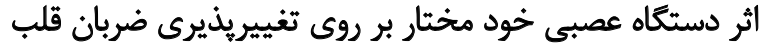

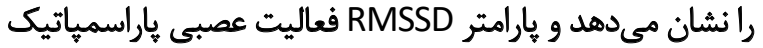

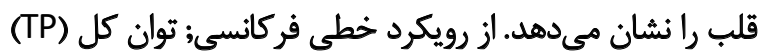

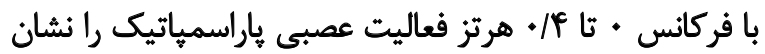

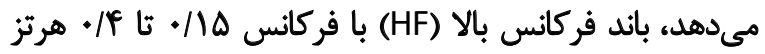

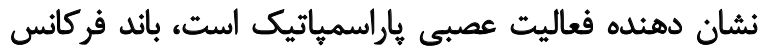

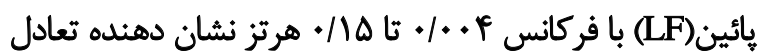

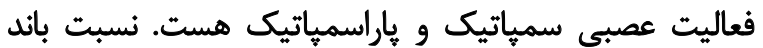

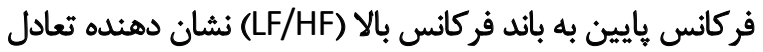

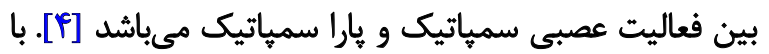

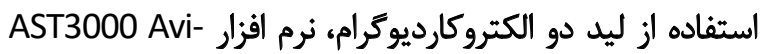

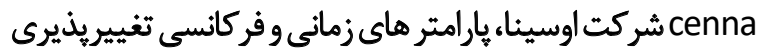

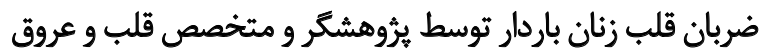
بررسى شدند.

به منظور بررسى جين دو لايه يوستى از جهار نقطه عضله

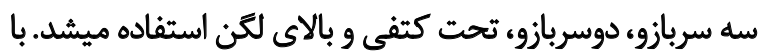

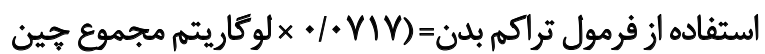

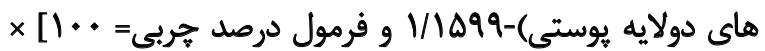

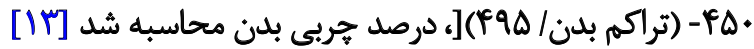

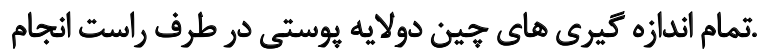

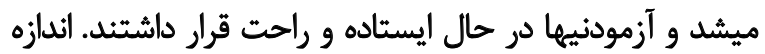

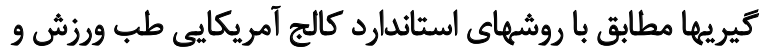

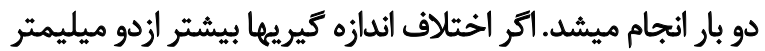

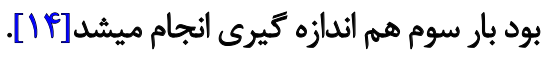

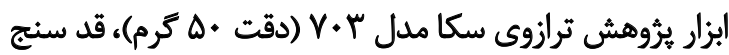

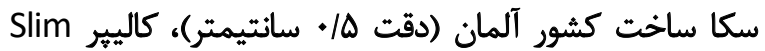
ساخت كشور استراليا (دقت يك ميليمتر)، فشارسنج

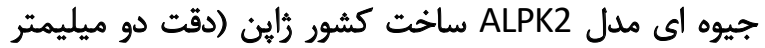

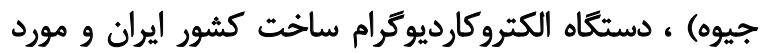

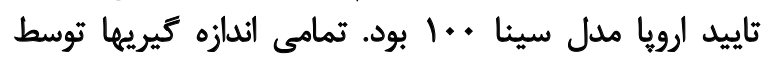
يك نفر و با وسايل اندازهكيرى يكسان انجام شد. هيك ليست

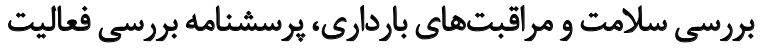

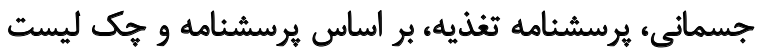

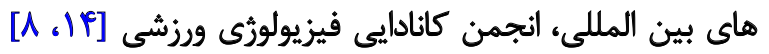

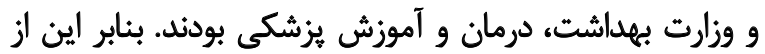

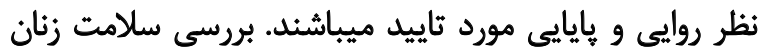

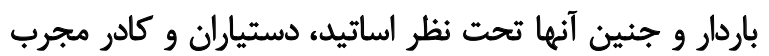
بيمارستان شهيد اكبرابادى تهران با تكميل فرم ها، معاينات و وانيا دارن 
جدولا. بروتكل تمرين تركيبى در كروه مداخله

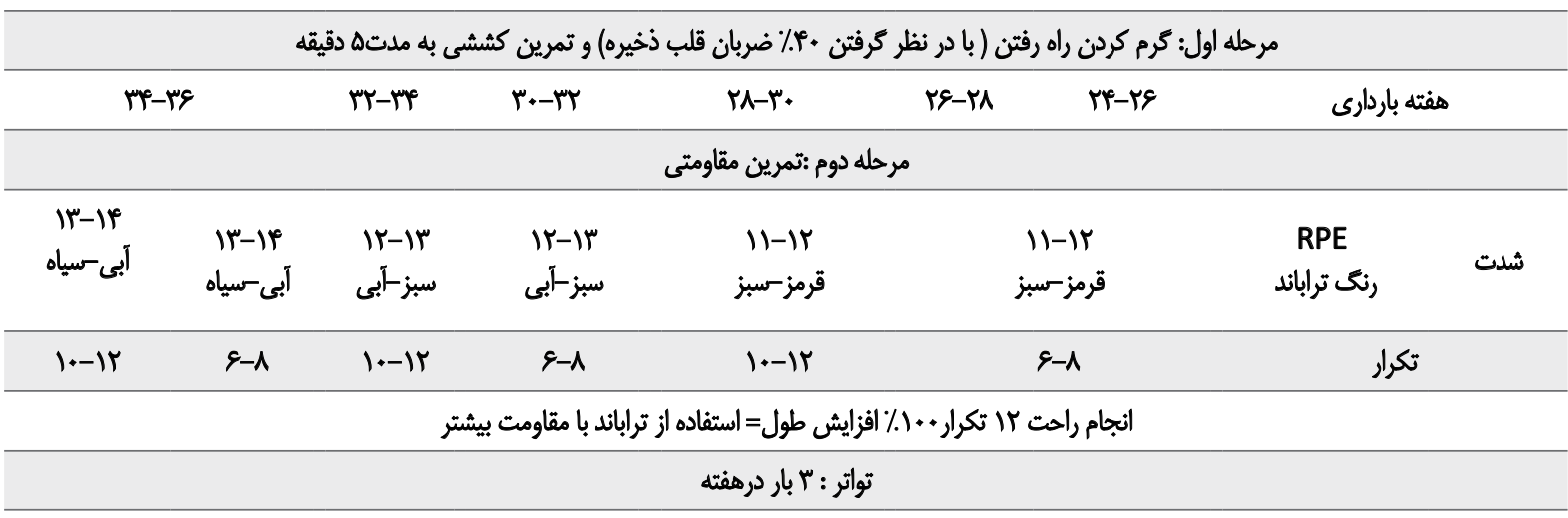

ست : r ست (قاصله ستها يك دقيقه و قاصله بين تمرينات مختلف ب دقيقه)

\begin{tabular}{|c|c|c|c|c|c|c|c|}
\hline \multicolumn{8}{|c|}{ نوع تمرين : سل نوع از تمرينات: فلكسيون شانه، ابداكشن شانه، فلكسيون واكستنسيون آرنج، اكستنسيون زانو، ابداكشن هيب، فلكسيون مج با. } \\
\hline \multicolumn{8}{|c|}{ مرحله سوم :تمرين هوازي ( بعد از دو دقيقه استراحت ) } \\
\hline $\begin{array}{l}1 \%-14 \\
1.8 .\end{array}$ & $\begin{array}{l}14-1 f \\
\% .\end{array}$ & $\begin{array}{l}\|,\|-\| \\
\% . \Delta .\end{array}$ & $\begin{array}{l}1 Y-\| \mu \\
\% \Delta .\end{array}$ & $\begin{array}{l}\|-\| r \\
\%\end{array}$ & $\begin{array}{l}\|-\| r \\
\%\end{array}$ & RPE\%HRR & شدت \\
\hline
\end{tabular}

هدت وتكرار: به مدت ه دقيقه سله بار در هفته

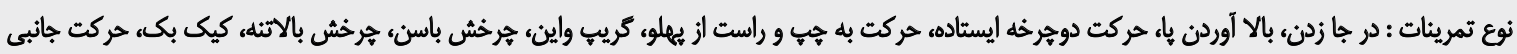

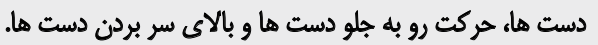

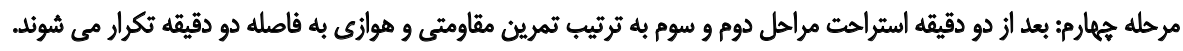

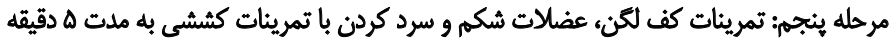

HRR ضربان قلب ذخيره و RPE: ميزان تلاش ادراك شده

كاهش Crp ميكردد . تمرين مستقيما توليد سيتوكاين از بافتهاى

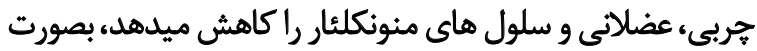

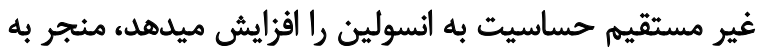

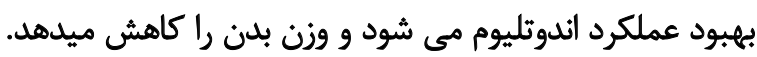

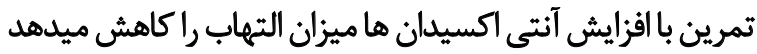

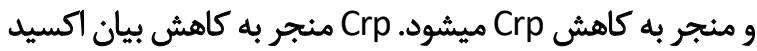

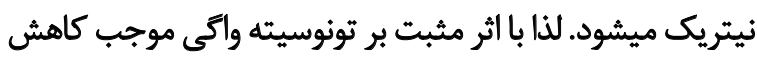

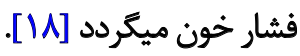

يارامتر SDNN نشان دهنده تعادل فعاليت عصبي سمياتيك

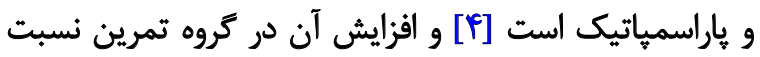

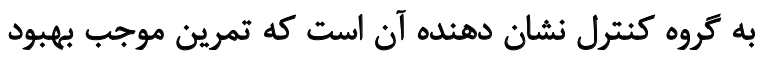

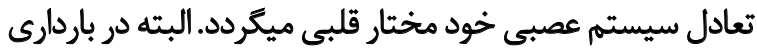

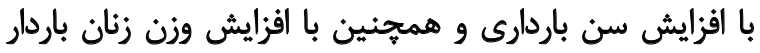

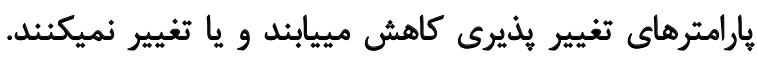

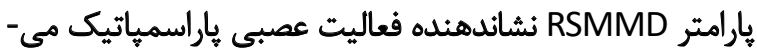

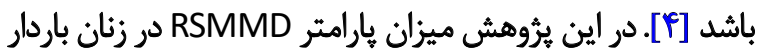

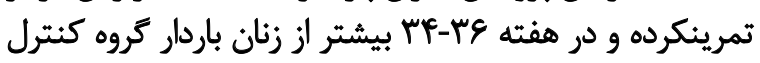
بود و اين تفاوت به لحاظ آمارى معنيدار بود. به عبان باردار كروه كيكر
كمتر بودن فشار خون و ضربان قلب در كروه تمرين نسبت به به اله

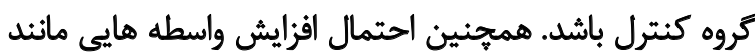

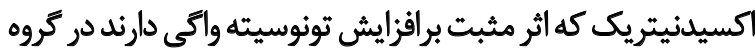

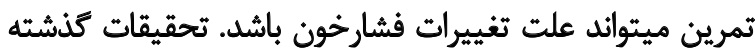

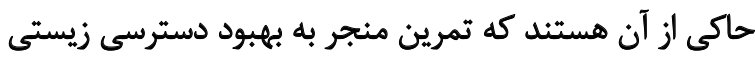

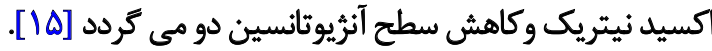

تحقيقات حاكى از آن هستند كه ميزان بالاى كراتين كينازب

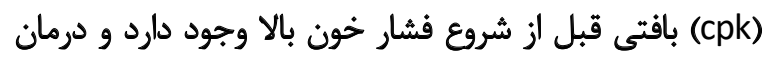

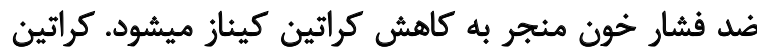

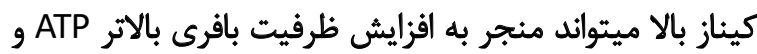

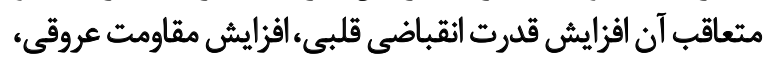

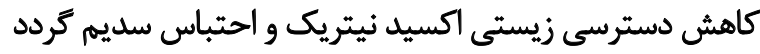

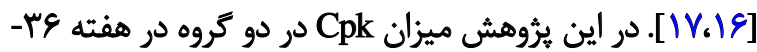

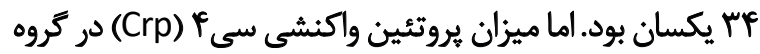

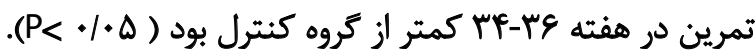

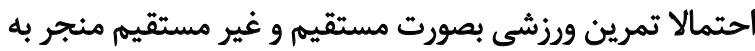

3. Creatine phosphokinase

4. C- Reactive protein 
جدول Y. ويثگتى هاى زنان باردار مراجعه كنثلده به بيمارستان شهيد اكبرآبادى تهران در هفته \&Y-YF باردارى

\begin{tabular}{|c|c|c|c|c|}
\hline \multirow{2}{*}{$\mathbf{P}$} & \multicolumn{2}{|c|}{ ميانكين ثانحراف معيار / تعداد (درصد) } & \multirow{2}{*}{\multicolumn{2}{|c|}{ ويؤَّى }} \\
\hline & توروه كثترل & كروه تمرين تركيبى & & \\
\hline$\cdot W^{*}$ & $r q / r+ \pm r / \Lambda$. & $Y N Y \cdot \pm F / A Y$ & \multicolumn{2}{|c|}{ سن (سال) } \\
\hline \multirow[t]{2}{*}{.$/ A r$} & $19 \pm 8 / 98$ & $198 . \pm F / 9 r$ & \multicolumn{2}{|c|}{ فعاليت فيزيكى (عاعت در هفته) } \\
\hline & $f(f \cdot)$ & $\Delta(\Delta \cdot)$ & 1 & \multirow{3}{*}{ تعلاد زايمان } \\
\hline \multirow{2}{*}{$+10 * *$} & $f(p+)$ & $p(p \cdot)$ & $r$ & \\
\hline & $r(r \cdot)$ & $1(1 \cdot)$ & $p$ & \\
\hline \multirow{2}{*}{$.10 * *$} & $\varepsilon(8 \cdot)$ & $V(V \cdot)$ & دييله وكمتر & \multirow{2}{*}{ سطح تحصيلات } \\
\hline & $f(p \cdot)$ & $r(r \cdot)$ & دانشكاهي & \\
\hline
\end{tabular}

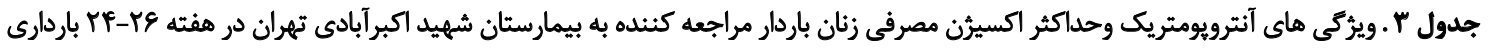

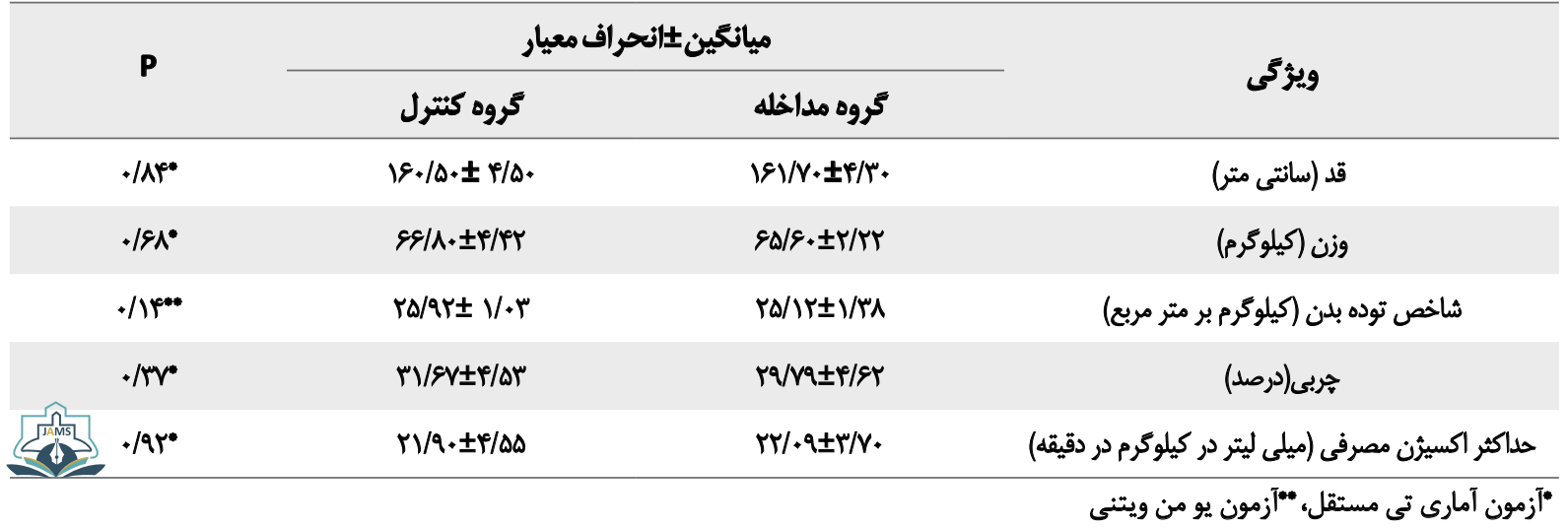

جدول F. جربان قلب و فشار خون زنان باردار قبل و بعد از تمرين تركيبى

\begin{tabular}{|c|c|c|c|c|c|c|}
\hline \multirow{3}{*}{ سطح معنى دارى } & \multirow{3}{*}{ سطح معنى دارى } & \multicolumn{4}{|c|}{ ميانكيندانحراف معيار } & \multirow{3}{*}{ آزمودنى ها } \\
\hline & & \multicolumn{2}{|c|}{ ئروه كثترل } & \multicolumn{2}{|c|}{ ئروه مداخله } & \\
\hline & & & MP & هist & هis & \\
\hline $1 . \Delta *$ & $.1 \cdot \Delta^{*}$ & $\begin{array}{l}\text { ضربه در دقيقه } 1 / M / M \\
\text { در/M }\end{array}$ & 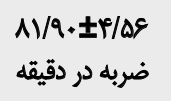 & 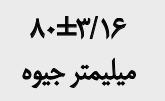 & 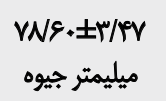 & ضربان قلب \\
\hline$+1+\cdot V^{* * * * *}$ & $<\cdot 1 \cdot * 1^{* * * *}$ & $\begin{array}{l}\text { VNIEY/OV } \\
\text { ميليمتر جيوه }\end{array}$ & $\begin{array}{l}M / f \cdot \pm / M \\
\text { ميليمتر جيوه }\end{array}$ & $\begin{array}{l}\text { ميليمتر جيوه } R / M / M \\
\text { ميوه }\end{array}$ & 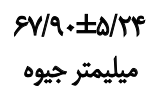 & دياستوليك \\
\hline$\cdot(\cdot r)^{* * * * *}$ & $<\cdot 1 \cdot \cdot 1^{* * *}$ & ميليمتر جيوه IN/FV & 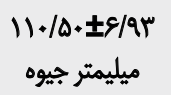 & ميليمتر جيوه & ميليمترجيوه & فيشار خُون \\
\hline
\end{tabular}

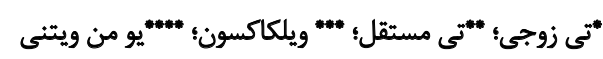




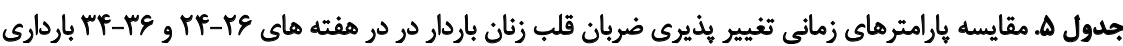

\begin{tabular}{|c|c|c|c|c|c|c|}
\hline \multicolumn{6}{|c|}{ ميانغين 土|نحرافمعيار } & \multirow{4}{*}{ 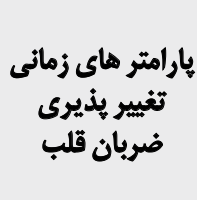 } \\
\hline \multicolumn{2}{|c|}{ سطح معنىدارى } & \multicolumn{4}{|c|}{ هفته باردارى } & \\
\hline \multirow{2}{*}{ كروه كثترل * } & \multirow{2}{*}{ كروه تمرين } & \multicolumn{2}{|c|}{ كروه تمرين } & \multicolumn{2}{|c|}{ كُوه كيترل } & \\
\hline & & re-m & $r \varepsilon-r$ & TE-m & rE-ry & \\
\hline.$/ 49$ & .1 .4 & $V E / T \pm V / T r$ & $g F / r \cdot \pm I r / I f$ & $\Delta V / \Lambda \cdot \pm I r / A V$ & $\varepsilon \Delta / \Delta \cdot \pm T \Delta / \varphi^{\prime} \varepsilon$ & $\begin{array}{l}\text { SDNN } \\
\text { هيلي ثائيه }\end{array}$ \\
\hline$+1+9$ & $\cdot / Q$ & $|F|+ \pm T Y / Q \mid$ & FNDFITV/+Y & $r V / V \cdot \pm r M / * 1$ & $\Delta F / 1 \cdot \pm F N \Delta r$ & $\begin{array}{l}\text { RMSSD } \\
\text { ميلي ثائيه }\end{array}$ \\
\hline
\end{tabular}

• آزمون آمارى ويلكاكسون sis

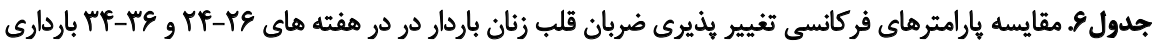

\begin{tabular}{|c|c|c|c|c|c|c|}
\hline & & \multicolumn{4}{|c|}{ مياتكين|نانحرافـمعيار } & \multirow{4}{*}{ هارامتر هاى تغيير يذيرى } \\
\hline & & \multicolumn{4}{|c|}{ هفته بار,دارى } & \\
\hline & & \multicolumn{2}{|c|}{ تروه تمرين } & \multicolumn{2}{|c|}{ ك عروه كتترل } & \\
\hline & & $r \varepsilon-m$ & $r \varepsilon-r T$ & & YE-Y & \\
\hline / & $\cdot M$ & 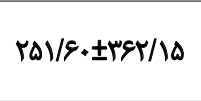 & $r \ldots / Y Y \pm P M q q$ & IYE/N. IIOT/AY & rAF士r|Q/SE & $\begin{array}{c}\text { LF } \\
\text { مجذور ميلى ثانيه }\end{array}$ \\
\hline .181 & +180 & $r++/ q+ \pm r q \varepsilon / \Delta q$ & $r 9 q / T \Delta \Delta \pm \Delta V N{ }^{\prime}+$ & $|r F / \Delta+ \pm|+\Delta / F \Delta$ & $\mid E Q / N I I F V / \Delta E$ & $\begin{array}{c}\text { HF } \\
\text { مجذور ميلى ثانيه }\end{array}$ \\
\hline 每 & $. / \Delta \mid$ & $r / * \lambda \pm r / * A$ & $r / \Delta q \pm r / r$. & $\mid / N A \pm r / r 1$ & $r / \Delta V \pm) / \& A$ & $\mathrm{LF} / \mathrm{HF}$ \\
\hline
\end{tabular}

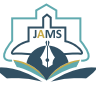

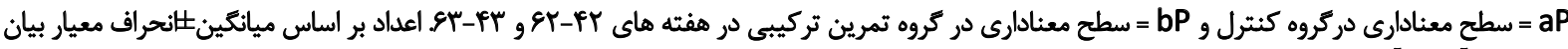
شده اند.** آزمون آمارى ويلكاكسون درون.

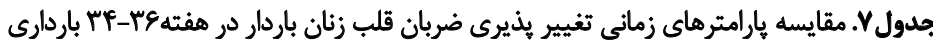

\begin{tabular}{|c|c|c|c|}
\hline \multirow{3}{*}{ سطح معنى دارى : } & \multicolumn{2}{|c|}{ ميانكين+|نحرافسمعيار } & \multirow{3}{*}{ متغير } \\
\hline & \multicolumn{2}{|c|}{ एะ-m } & \\
\hline & كروه كثترل هفته & كروه تمرين هفته & \\
\hline $.1 . .1$ & $\Delta / / A \cdot \pm 1 r / A V$ & $V E / T \pm V / T r$ & $\begin{array}{l}\text { SDNN } \\
\text { ميلى ثائيه }\end{array}$ \\
\hline $1 . \varphi$ & $r V / N+ \pm r M /+1$ & $P N|\cdot \pm T Y| F \mid$ & $\begin{array}{l}\text { RMSSD } \\
\text { ميلى ثانيه }\end{array}$ \\
\hline
\end{tabular}

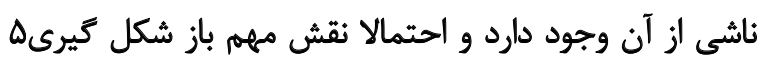

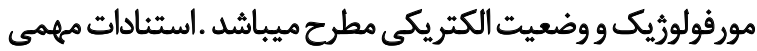

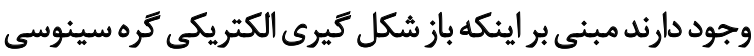
با تمرين تحريك ميكردد [19].
تمرين موجب برترى نسبى فعاليت عصبى ياراسمياتيك در تروه

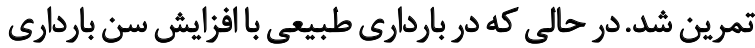

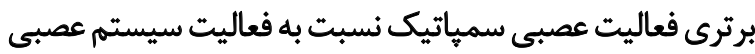

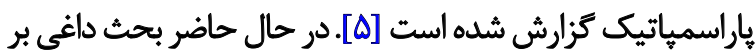

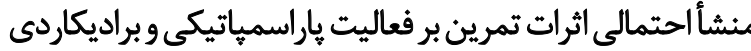


جدول A. مثايسه يارامترهاى فركائسى تغيير يذيرى ضربان قلب زئن باردار در هفته عr-Mf باردارى

\begin{tabular}{|c|c|c|c|}
\hline \multirow{3}{*}{ سطح معنى دارى * } & \multicolumn{2}{|c|}{ مياتيّين+انحراقمعيار } & \multirow{3}{*}{ متغير } \\
\hline & \multicolumn{2}{|c|}{$r \varepsilon-r$} & \\
\hline & كروه كتثرل هفته & كُوره تمرين هفته & \\
\hline.$/ 97$ & ITEN. EIAT/AY & 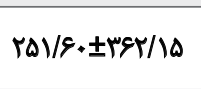 & $\begin{array}{c}\text { LF } \\
\text { مجذور ميلى ثانيه }\end{array}$ \\
\hline .199 & $\mid \pi / \Delta \cdot \pm 1 \cdot \Delta / r \Delta$ & $r+. / q+ \pm r q \varepsilon / 0 q$ & $\begin{array}{c}\text { HF } \\
\text { مجذور ميلى ثانيه }\end{array}$ \\
\hline.$/ 4 \Delta$ & $\mid / R \wedge \pm r / r)$ & $r /+\Lambda \pm r /+1$ & LF/HF \\
\hline
\end{tabular}

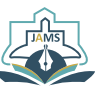

قلب زنان باردار در زمان استراحت در هفتههاى مختلف باردارى

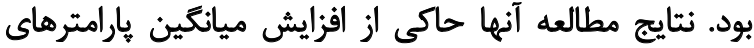

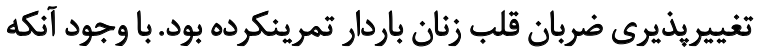

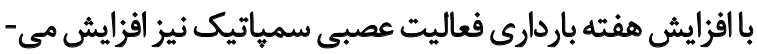

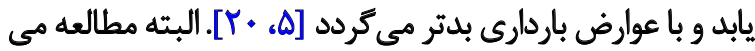

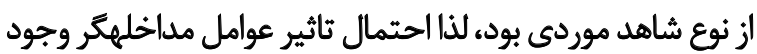

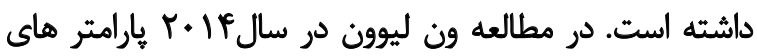

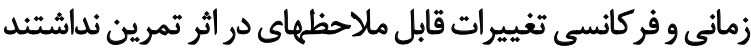

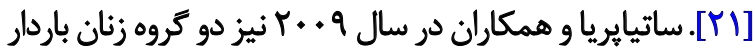

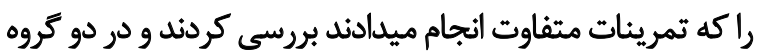

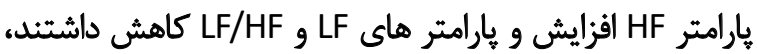

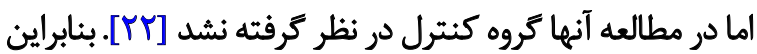

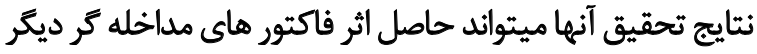

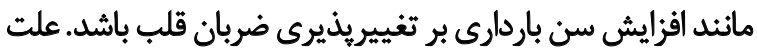

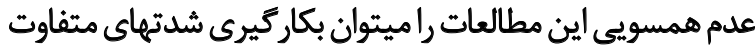

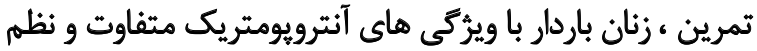

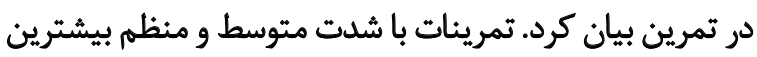

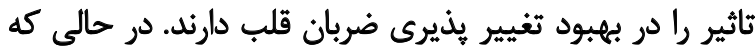

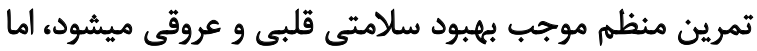

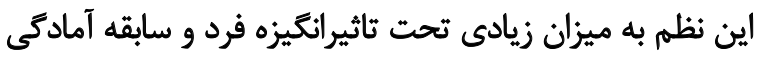

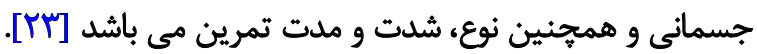

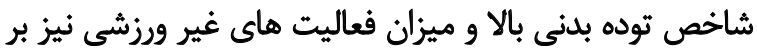
نتايج تحقيق موثر ميباشند.

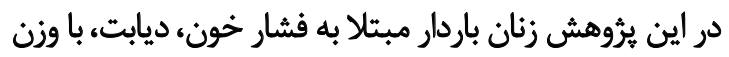

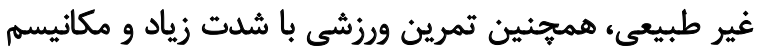

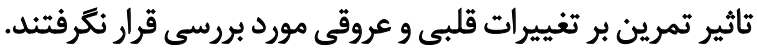

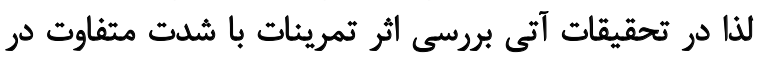

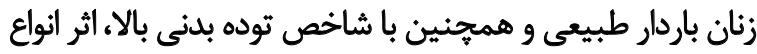

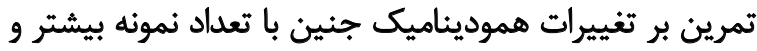

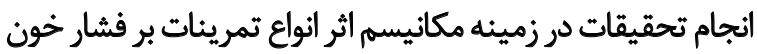
و تغيير بذيرى ضربان قلب سودمند هستند.

اعداد بر اساس انحراف معيارثميانكين بيان شده اند.تآزمون آمارى يو من ويتنى

نكته قوت اين بروهش وجود كروه كنترل، مقايسه كروه مداخله

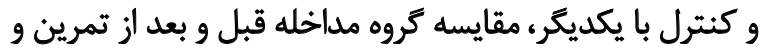

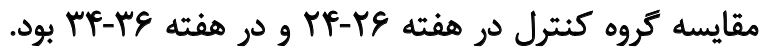

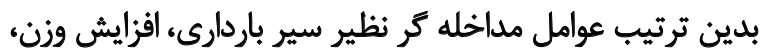

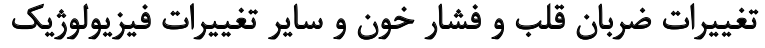

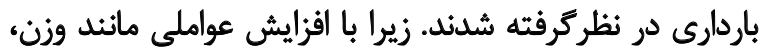

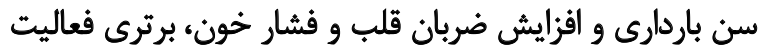
عصبى سمياتيكى بر تغييريذيرى ضرئي

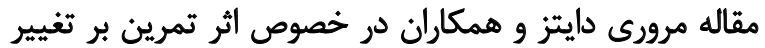

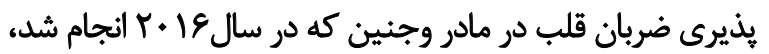

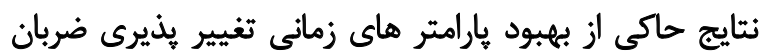

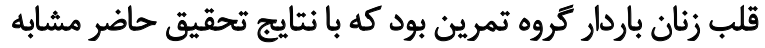

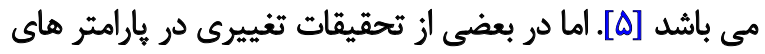

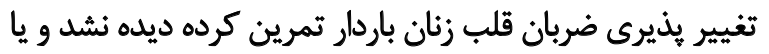

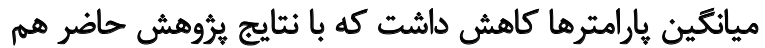

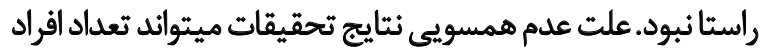

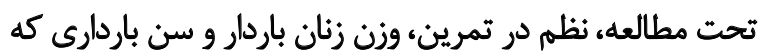

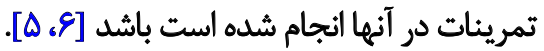
در يروهش حاضر تعداد ضربان قلب زنان باردار بصورت قابل

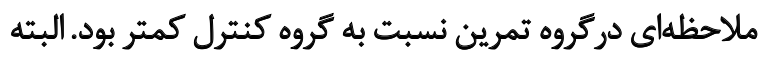

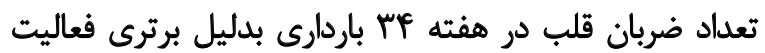

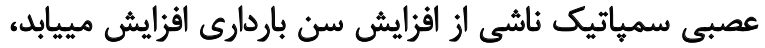

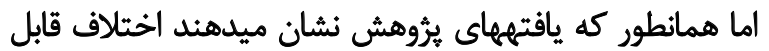

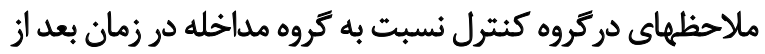

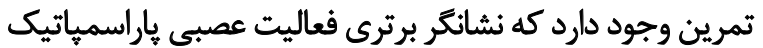

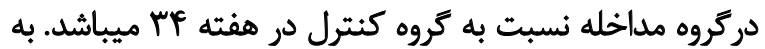

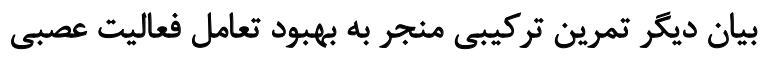

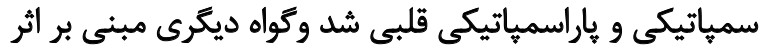
تمرين بر ارتقاء فعاليت سيستم عصبى خود مختار قلب ميكي ميباشد.

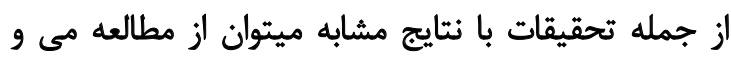

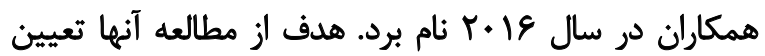
تاثير تمرين ورزشى بر بهبود عملكرد سيستم عصبى خود مختار 


\section{نتيجه كيرى}

در مجموع در يُروهش حاضر نشان داده شد كه تمرين تركيبى

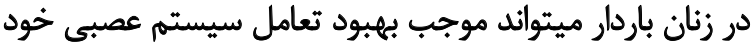

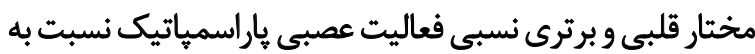

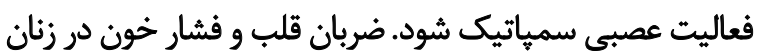

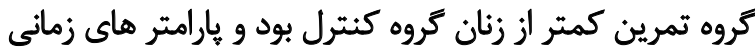

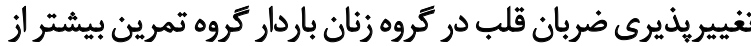

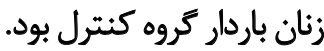
مالاحظات اخلاقى

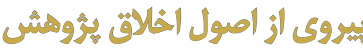

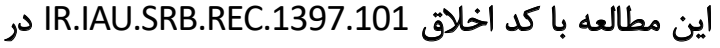

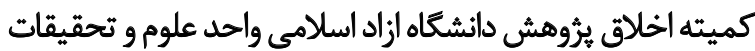

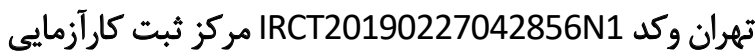

$$
\text { بالينى به ثبت رسيد. }
$$

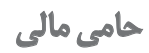

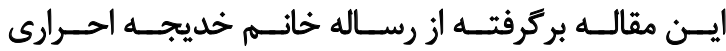

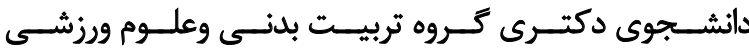

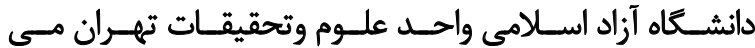
باشــد.

\section{مشار كت نويسيناك انان}

تمامى نويسندكان معيارهاى استاندارد نويسندكى بر اساس

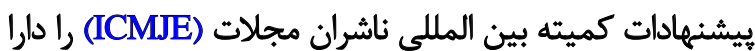
بودند.

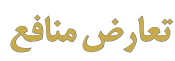

بدين وسيله نويسندكان تصريح مى نمايند كه هيجكونه تضاد مثافعى در خصوص بروهش حاضر وجود ندارد. تصني

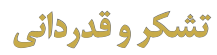

نويسندكان مراتب تقديروتشكر خود را از اساتيد، يزشكان،

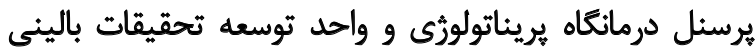

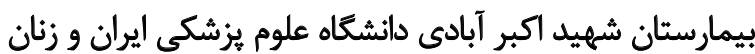

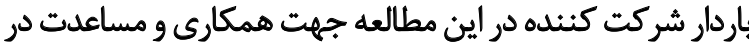
طول مطالعه اعلام ميدارند. 


\section{References}

[1] Hanson M, Gluckman P. Developmental origins of health and diseaseglobal public health implications. Best Pract Res Clin Obstet Gynaecol. 2015; 29(1):24-31. [DOI:10.1016/j.bpobgyn.2014.06.007] [PMID]

[2] Perales M, Artal R, Lucia A. Exercise during pregnancy. JAMA. 2017 21(11):1113-4. [DOI:10.1001/jama.2017.0593] [PMID]

[3] May LE, Allen JJ, Gustafson KM. Fetal and maternal cardiac responses to physical activity and exercise during pregnancy. Early Hum Dev. 2016; 1(4):49-52. [DOI:10.1016/j.earlhumdev.2016.01.005] [PMID]

[4] Davis G, Graham K, Michael S. Cardiac autonomic responses during exercise and post-exercise recovery using heart rate variability and systolic time intervals-a review. systolic time intervals-a review. Front Physiol. 2017; 8(7):301. [DOI:10.3389/fphys.2017.00301] [PMID] [PMCID]

[5] Dietz P, Watson ED, Sattler MC, Ruf W, Titze S, van Poppel M. The influence of physical activity during pregnancy on maternal, fetal or infant heart rate variability: A systematic review. BMC Pregnancy Childbirth. 2016; 16(1):326.6. [DOI:10.1186/s12884-016-1121-7] [PMID] [PMCID]

[6] Roldan-Reoyo O, Pelaez M, May L, Barakat R. Influence of materna physical exercise on fetal and maternal heart rate responses. Ger J Exerc Sport Res. 2019; 5(2) 1-8.

[7] O'Connor PJ, Poudevigne MS, Cress ME, Motl RW, Clapp JF. Safety and efficacy of supervised strength training adopted in pregnancy. J Phys Act Health. 2011; 8(3):309-20. [DOI:10.1123/jpah.8.3.309] [PMID]

[8] Smith KM, Campbell CG. Physical activity during pregnancy: Impact of applying different physical activity guidelines. J Pregnancy. 2013; 2013: 165617. [DOI:10.1155/2013/165617] [PMID] [PMCID]

[9] VanVoorhis CW, Morgan BL. Understanding power and rules of thumb for determining sample sizes. Tutor Quant Methods Psychol. 2007; 3(2):43-50. [DOI:10.20982/tqmp.03.2.p043]

[10] Gregg VH, Ferguson JE. Exercise in pregnancy. Clin Sports Med. 2017; 36(4):741-52. [DOI:10.1016/j.csm.2017.05.005] [PMID]

[11] Karvonen MJ, Kentala E, Mustala O. The effects of training on heart rate: A longitudinal study. Ann Med Exp Biol Fenn. 1957; 35(1):307-15.

[12] Sammito S, Böckelmann I. Factors influencing heart rate variability. Int Cardiovasc Forum Journal. 2016; 4(6):321-5. [DOI:10.17987/icfj. v6i0.242]

[13] Durnin JV, Womersley JV. Body fat assessed from total body density and its estimation from skinfold thickness: Measurements on 481 men and women aged from 16 to 72 years. Br J Nutr. 1974; 32(1):77-97. [DOI:10.1079/BJN19740060] [PMID]

[14] Gibson AL, Wagner D, Heyward V. Advanced Fitness Assessment and Exercise Prescription, $8^{\text {th }}$ ed. Champaign: Human kinetics; 2018.

[15] Nystoriak MA, Bhatnagar A. Cardiovascular effects and benefits of exercise. Front Cardiovasc Med. 2018; 5(1):135. [DOI:10.3389/ fcvm.2018.00135] [PMID] [PMCID]

[16] Karamat FA, Oudman I, Haan YC, van Kuilenburg AB, Leen R, Danser JA, et al. Creatine kinase inhibition lowers systemic arterial blood pressure in spontaneously hypertensive rats: A randomized controlled trial. J Hypertens. 2016; 34(12):2418-26. [DOI:10.1097/HJH.0000000000001090] [PMID]

[17] Sukul S, Bahinipati J, Patra S, Ravichandran K. Serum creatine kinase activity among hypertensive patients and its role as a predictor for failure of antihypertensive treatment. Journal of Clinical \& Diagnostic Research. 2018; 12(11):121-5.
[18] Kasapis C, Thompson PD. The effects of physical activity on serum C-reactive protein and inflammatory markers: A systematic review. J Am Coll Cardiol. 2005; 45(10):1563-9. [DOI:10.1016/j.jacc.2004.12.077] [PMID]

[19] Boyett MR, Wang Y, Nakao S, Ariyaratnam J, Hart G, Monfredi O, et al. Point: Exercise training-induced bradycardia is caused by changes in intrinsic sinus node function. J Appl Physiol. 2017; 123(3):684-5 [DOI:10.1152/japplphysiol.00604.2017] [PMID] [PMCID]

[20] May LE, Knowlton J, Hanson J, Suminski R, Paynter C, Fang X, et al. Effects of exercise during pregnancy on maternal heart rate and heart rate variability. PMR. 2016; 8(7):611-7. [DOI:10.1016/j.pmrj.2015.11.006] [PMID]

[21] Van Leeuwen P, Gustafson KM, Cysarz D, Geue D, May LE, Grönemeye $D$. Aerobic exercise during pregnancy and presence of fetal-maternal heart rate synchronization. PLOS One. 2014; 9(8):103-6. [DOI:10.1371/ journal.pone.0106036] [PMID] [PMCID]

[22] Satyapriya M, Nagendra HR, Nagarathna R, Padmalatha V. Effect of integrated yoga on stress and heart rate variability in pregnant women. Int J Gynaecol Obstet. 2009; 104(3):218-22. [DOI:10.1016/j. ijgo.2008.11.013] [PMID]

[23] Silva JR, Rumpf MC, Hertzog M, Castagna C, Farooq A, Girard O, et al. Acute and residual soccer match-related fatigue: A systematic review and meta-analysis. Sports Med. 2018; 48(3):539-83. [DOI:10.1007/ s40279-017-0798-8] [PMID] 